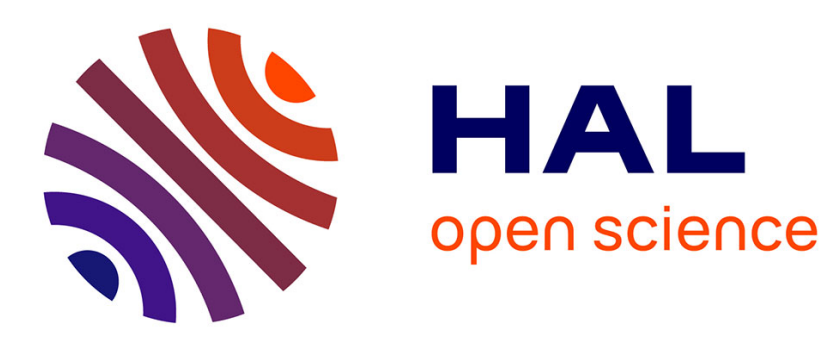

\title{
Analysis of heat sources accompanying the fatigue of 2024 T3 aluminium alloys
}

\author{
A. E. Morabito, Andre Chrysochoos, V. Dattoma, U. Galietti
}

\section{To cite this version:}

A. E. Morabito, Andre Chrysochoos, V. Dattoma, U. Galietti. Analysis of heat sources accompanying the fatigue of 2024 T3 aluminium alloys. International Journal of Fatigue, 2007, 29, pp.977-984. 10.1016/j.ijfatigue.2006.06.015 . hal-00572433

\section{HAL Id: hal-00572433 \\ https://hal.science/hal-00572433}

Submitted on 23 Sep 2021

HAL is a multi-disciplinary open access archive for the deposit and dissemination of scientific research documents, whether they are published or not. The documents may come from teaching and research institutions in France or abroad, or from public or private research centers.
L'archive ouverte pluridisciplinaire HAL, est destinée au dépôt et à la diffusion de documents scientifiques de niveau recherche, publiés ou non, émanant des établissements d'enseignement et de recherche français ou étrangers, des laboratoires publics ou privés. 


\title{
Analysis of heat sources accompanying the fatigue of 2024 T3 aluminium alloys
}

\author{
A.E. Morabito ${ }^{\text {a,* }}$, A. Chrysochoos ${ }^{\text {b }}$, V. Dattoma ${ }^{a}$, U. Galietti ${ }^{\text {c }}$ \\ a Dipartimento di Ingegneria dell'Innovazione, Università di Lecce, via per Arnesano 73100 Lecce, Italy \\ ${ }^{\mathrm{b}}$ Laboratoire de Mécanique et Génie Civil, Université Montpellier II (France) 860, route de St Priest 34090 Montpellier, France \\ ${ }^{\mathrm{c}}$ Dipartimento di Ingegneria Meccanica e Gestionale, Politecnico di Bari, Viale Japigia 18270126 Bari, Italy
}

\begin{abstract}
This paper deals with the calorimetric analysis of fatigue mechanisms of an aluminium alloy. Local 1D and 2D expressions of the heat diffusion equation were used to separately estimate the coupling and dissipative sources accompanying the fatigue test. The image processing was based on local approximations of surface temperature fields provided by an IR camera. The set of approximation functions accounted for the spectral properties of the sought sources. The analysis of thermoelastic source distribution allowed us to point out the possible heterogeneous character of fatigue loading from the beginning of the test. We observed that the zones where thermoelastic source amplitudes were the highest were potential loci of fatigue crack onset. On the other hand, such fine results on the dissipative source distribution were hard to obtain because of low signal-to-noise ratios. Nevertheless, we observed that the global dissipated energy rates increased linearly with the loading frequency.
\end{abstract}

Keywords: Aluminium alloy; Calorimetric analysis; Thermal images; Thermoelastic sources; Fatigue dissipation

\section{Introduction}

Fatigue characterization of materials and mechanical components requires statistical processing of numerous time-consuming and expensive mechanical cyclic tests. This problem is even greater for aluminium alloys since the Wöhler curve construction does not generally show asymptotic stress classically associated with fatigue limits.

Alternative experimental approaches were developed to rapidly provide reliable fatigue characteristics. Among these, thermal methods based on the analysis of self-heating during a stepwise loading fatigue test must be mentioned [1-4]. The authors of the latter studies claim that the remarkable change in the heating regime, observed within a certain stress range, is empirically related to the fatigue limit of the material. Although realistic estimates

\footnotetext{
* Corresponding author. Tel.: +39 0832297249; fax: +390832297279.

E-mail address: annaeva.morabito@unile.it (A.E. Morabito).
}

of this limit were sometimes obtained, these thermal approaches often led to questionable results. Indeed, the physical meaning of the threshold stress evaluated by these thermal methods is not very clear. In [5], it was shown that this stress is not actually the fatigue limit since it is closely related to the appearance of localized plastic phenomena (persistent slip bands) and not to the crack onset.

In some previous works, it was shown that the direct use of temperature specimens as fatigue indicators is not always reliable because the temperature variation is not totally intrinsic to the material behavior [6]. It actually depends on the diffusion properties (material effect) but also on the thermal boundary conditions and the heat source distribution (structure effects).

In this work, fatigue phenomena are therefore studied using a calorimetric approach - the aim is to assess the different heat sources accompanying the fatigue test. The application of this approach to aluminium alloys is particularly tricky because of their high thermal diffusivity. 
The regularizing effect of heat diffusion actually limits temperature variations and flattens the thermal gradients.

Heat sources were derived from thermal images provided by an infrared camera using a local expression of the heat equation. A specific thermal image processing procedure was developed to separately estimate the dissipated energy coming from the irreversible variations in the microstructural defects and from the thermoelastic coupling sources induced by the reversible thermal expansion of the crystalline network.

The paper is laid out as follows: Section 2 is devoted to the description of the theoretical tools used to construct the local heat equation, define the different heat sources and discuss the $1 \mathrm{D}$ and 2D thermal diffusion models. Section 3 describes the experimental setup. Section 4 shows the image processing method together with some numerical simulations in order to illustrate the consistency but also the limits of the heat source computation in cases of noisy, under-sampled thermal signals. Finally, some experimental results are shown in Section 5 for the aluminium alloy $2024 \mathrm{~T} 3$ considered here.

\section{Theoretical tools}

From mechanical and thermodynamic standpoints, fatigue was considered as a dissipative, quasi-static process. The Thermodynamics of Irreversible Process concept was the thermomechanical framework used to interpret the experimental results - this concept is based on the local state axiom and consequently characterizes the equilibrium state of each volume material element through a set of $N$ state variables. The selected variables are the absolute temperature $T$, the linearized strain tensor $\varepsilon$ and $N-2$ scalar components $\alpha_{1}, \alpha_{2}, \ldots, \alpha_{\mathrm{N}-2}$ of the vector $\alpha$ gathering the internal variables. These latter describe the macroscopic effects of complex and often coupled microstructural phenomena.

Using the generalized standard materials formalism it is possible to sum-up the material thermomechanical behavior by means of a thermodynamic potential and a dissipation potential. By construction, the thermodynamic potential is the specific Helmholtz free energy $\Psi\left(T, \varepsilon, \alpha_{\mathrm{i}}\right)$ and the dissipation potential is a function of the state variable fluxes, i.e. $\varphi\left(\vec{q}, \dot{\varepsilon}, \dot{\alpha}_{i} ; T\right)$, with $\vec{q}$ being the heat influx and $T$ acting as a parameter [7].

Combining the first and second principles of thermodynamics leads to the following local heat equation:

$$
\begin{aligned}
\rho C \dot{T}-\operatorname{div}(K: & \operatorname{grad} T) \\
= & \underbrace{\left(\sigma-\rho \psi_{, \varepsilon}\right): \dot{\varepsilon}-\rho \psi_{, \alpha}: \dot{\alpha}}_{d_{1}} \\
& +\underbrace{\rho T \psi_{, T \varepsilon}: \dot{\varepsilon}+\rho T \psi_{, T \alpha}: \dot{\alpha}}_{s_{\mathrm{the}}+s_{\mathrm{ic}}}+r_{\mathrm{ext}}
\end{aligned}
$$

where $\rho$ is the mass density, $C$ the specific heat, $K$ the material conduction tensor, and $\sigma$ the Cauchy stress tensor.

The left-hand side of Eq. (1) is a differential operator applied to $T$, while the right-hand side groups all possible heat sources accompanying the deformation process. They are in turn: the intrinsic dissipation $d_{1}$, the thermomechanical coupling sources consisting of the thermoelastic source $s_{\text {the }}$ and the other internal coupling sources $s_{\mathrm{ic}}$, and the external volume heat supply $r_{\text {ext }}$.

The overall heat source can then be theoretically estimated by evaluating the left-hand side of heat equation. Nevertheless, the thermal information given by the IR camera only concerns surface temperature fields. The heat source evaluation throughout the specimen gauge part (3D inverse problem) is an ill-posed problem that is impossible to solve without any information on the heat source distribution [8]. If thermal gradients remain small throughout the thickness of the specimen (Fig. 1), a depthwise averaged heat source distribution can be still be estimated [9].

To perform the heat source evaluation, the following hypotheses were formulated:

- the heat conduction is isotropic and the related coefficient $k$ remains constant during the test;

- the density $\rho$ and the specific heat $C$ are material constants, independent of the internal state;

- the convective terms included in the material time derivation are neglected;

- the external heat supply $r_{\text {ext }}$ (here only due to radiation heat exchanges) is time independent. Consequently, the equilibrium temperature field $T_{0}$ verifies the equation:

$-k \Delta T_{0}=r_{\mathrm{ext}}$

The temperature variations $\theta=T-T_{0}$ induced by fatigue mechanisms are too small to have any influence on the microstructural state. It is then possible to state that $s_{\mathrm{ic}}=0$. Under these hypotheses, the local heat Eq. (1) can be rewritten in the following simplified form:

$\rho C \frac{\partial \theta}{\partial t}-k \Delta \theta=d_{1}+s_{\text {the }}=s$

where $s=d_{1}+s_{\text {the }}$ is henceforth the overall volume heat source $s$.

By integrating Eq. (3) over the thickness and assuming that the measured surface thermal map is very close to the depthwise averaged temperature field $\theta(x, y, t)$, the following equation can be obtained:

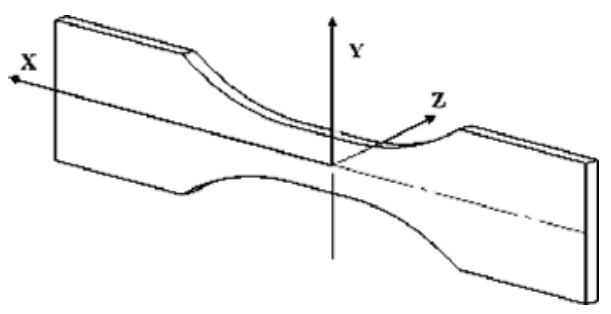

Fig. 1. Shape of the specimen. 
$\rho C\left(\frac{\partial \theta}{\partial t}+\frac{\theta}{\tau_{\mathrm{th}}^{2 \mathrm{D}}}\right)-k\left(\frac{\partial^{2} \theta}{\partial x^{2}}+\frac{\partial^{2} \theta}{\partial y^{2}}\right)=s$

where $s=s(x, y, t)$ is the 2D heat source distribution and $\tau_{\mathrm{th}}^{2 \mathrm{D}}$ is a time constant that characterizes the perpendicular heat exchanges (by convection and radiation) between the front and back specimen faces and the surroundings. This 2D-approach has already been successfully used to track localization zones during monotonic tensile tests [10].

The next possibility is to consider a one-dimensional (1D) diffusion problem, which leads to an estimate of the mean heat source over each sample cross-section $S$ (Fig. 1). The integration of Eq. (3) over $S$ leads to

$\rho C\left(\frac{\partial \theta}{\partial t}+\frac{\theta}{\tau_{\mathrm{th}}^{\mathrm{lD}}}\right)-k\left(\frac{\partial^{2} \theta}{\partial x^{2}}\right)=s$

where the mean temperature of the cross-section $\theta(x, t)$ is identified with the surface temperature, and $s=s(x, t)$ is the longitudinal heat source profile. Moreover, the time constant $\tau_{\text {th }}^{1 \mathrm{D}}$ characterizes lateral heat exchanges by conduction, convection and radiation between the specimen and the surroundings. These exchanges are supposed to be proportional to the temperature variation (linear Fourier boundary conditions).

In order to estimate the left-hand side of Eq. (5), longitudinal temperature profiles were built by averaging the surface temperature over the specimen width.

\section{Experimental setup}

The material under examination is the aluminium alloy 2024 T3. The fatigue tests were conducted using an MTS servo-hydraulic machine equipped with a $\pm 25 \mathrm{kN}$ load cell. Thin flat specimens were used with a gauge part volume equal to $3 \times 15 \times 10 \mathrm{~mm}^{3}$ (Fig. 2).

The experimental setup included a focal plane array infrared camera (IRFPA Cedip MW). The maximum frame-rate of the IR camera was 250 images per second, with each image consisting of $64 \times 120$ pixels.

During the tests, the infrared device was placed in front of the specimen, with the lens axis being fixed and perpendicular to the specimen surface. The spatial resolution was approximately $0.13 \mathrm{~mm} /$ pixel.

The fatigue tests were carried out by imposing a series of loading blocks (Fig. 3).
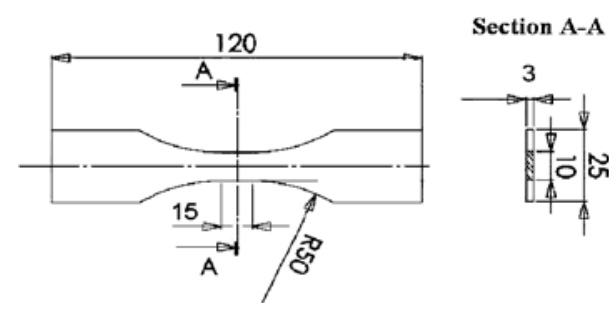

Fig. 2. Specimen drawing.

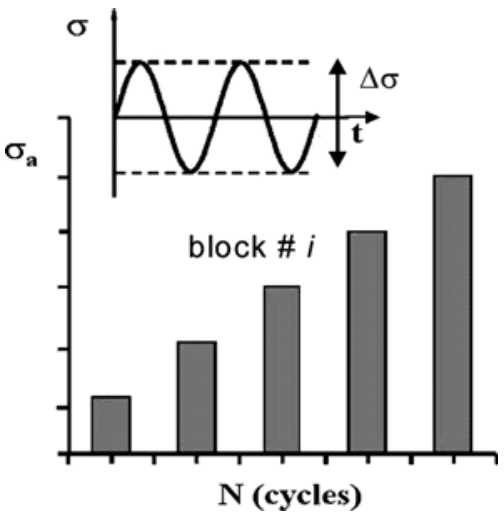

Fig. 3. Stepwise loading procedure: blocks of 20,000 cycles.

Each block consisted of 20,000 cycles performed at constant loading frequency $f_{\mathrm{L}}$, constant load ratio $R_{\sigma}$, and constant stress range $\Delta \sigma$.

At the end of each loading block, the thermal equilibrium was established before the next block started.

\section{Image processing}

The construction of the heat source distribution via Eqs. (4) and (5) requires the evaluation of partial derivative operators applied to noisy digital signals.

To improve the signal-to-noise ratio, it is then necessary to reduce the noise amplitude without modifying the thermal gradients. Among several possible methods, a special local least-squares fitting of the thermal signal was considered in this work. The approximation function accounts for the spectral properties of the sought sources.

The linearity of Eqs. (4) and (5) and that of the respective boundary conditions enabled us to separately analyze the influence of thermoelastic and dissipative heat sources. $\theta_{\text {the }}$ and $\theta_{\mathrm{d}}$ respectively represented the thermoelastic and dissipative components of the temperature signal, with the thermoelastic behavior of the aluminium assumed to be linear and isotropic.

\subsection{Heat source spectral properties}

For a pure uniaxial, sinusoidal elastic loading, $\sigma(t)=$ $\sigma_{\mathrm{m}}+\frac{\Delta \sigma}{2} \sin \left(2 \pi f_{\mathrm{L}} t\right)$ defined by a mean stress $\sigma_{\mathrm{m}}$, a stress range $\Delta \sigma$ and a loading frequency $f_{\mathrm{L}}$, we can show that the thermoelastic source is written as

$s_{\text {the }}=\rho T_{\psi, T \varepsilon}: \dot{\varepsilon}=-T \lambda\left[\dot{\sigma}+9 K_{0} \lambda \dot{\theta}\right]$

where $\lambda$ is the linear thermal expansion coefficient and $K_{0}$ is the elastic bulk modulus in hydrostatic compression.

Combining Eq. (6) with Eq. (5) (or Eq. (4)), we then obtain that

$\rho C\left(\dot{\theta}+\frac{\theta}{\tau_{\mathrm{th}}^{1 \mathrm{D}}}\right)-k\left(\frac{\partial^{2} \theta}{\partial x^{2}}\right)=-T \lambda\left[\dot{\sigma}+9 K_{0} \lambda \dot{\theta}\right]$

and therefore 


$$
\left(1+\frac{9 K_{0} \lambda^{2} T}{\rho C}\right) \dot{\theta}+\left(\frac{\theta}{\tau_{\mathrm{th}}^{\mathrm{iD}}}\right)-\frac{k}{\rho C}\left(\frac{\partial^{2} \theta}{\partial x^{2}}\right)=-\frac{T \lambda}{\rho C} \dot{\sigma}
$$

For temperature $T$ close to the equilibrium temperature $T_{0}$, we get

$\frac{9 K_{0} \lambda^{2}}{\rho C} T \approx \frac{9 K_{0} \lambda^{2}}{\rho C} T_{0} \ll 1$

It is then possible to rewrite the thermoelastic source as

$s_{\text {the }}=-T_{0} \lambda \dot{\sigma}$

In such conditions, it is easy to verify that

- the thermoelastic source has the same frequency spectrum as the stress signal;

- the variation in thermoelastic energy $w_{\text {the }}$ vanishes at the end of each loading cycle, so that we get

$w_{\text {the }}=\oint_{\text {Cycle }} s_{\text {the }} \mathrm{d} \tau=0$

Regarding the dissipative effects, we considered that the dissipation averaged over a whole number $n$ of complete cycles $(n \sim 50)$ :

$\bar{d}_{1}=\oint_{n \text { cycles }} n^{-1} f_{\mathrm{L}} d_{1} \mathrm{~d} \tau$

may characterize the slow degradation of the material microstructure due to fatigue phenomena. The average dissipation per cycle $\bar{d}_{1}$ is thus a positive heat source whose spectrum is limited to very low frequencies.

By denoting $\Delta s_{\text {the }}$ as the range of the thermoelastic source averaged over $n$ cycles, the aim of image processing is to separately assess $\Delta s_{\text {the }}$ and $\bar{d}_{1}$.

\subsection{Fitting}

The local fitting function of the temperature charts is defined by

$$
\begin{aligned}
\theta_{\text {mod }}(\vec{x}, t)= & p_{1}(\vec{x}) t+p_{2}(\vec{x})+p_{3}(\vec{x}) \cos \left(2 \pi f_{\mathrm{L}} t\right) \\
& +p_{4}(\vec{x}) \sin \left(2 \pi f_{\mathrm{L}} t\right)
\end{aligned}
$$

where the trigonometric time functions describe the periodic part of the thermoelastic effects while the linear time function takes transient effects due to heat losses, dissipative heating and possible drifts in the equilibrium temperature into account. The analytical expression of functions $p_{i}(\vec{x}), i=1, \ldots, 4$ are

- second-order polynomials in $x$ and $y$ for the 2D model (Eq. (4));

- second-order polynomials in $x$ for the 1D model (Eq. (5)).

These polynomials enable us to account for the possible space homogeneity of the source pattern.

The efficiency of the local data fitting can be illustrated by the temperature difference between the noisy measure- ments and the fitted values. In 1D (profile) processing, the mean absolute error was typically about $0.01^{\circ} \mathrm{C}$, while the standard deviation was $0.007{ }^{\circ} \mathrm{C}$.

The relative error naturally depends on the temperature level. In the present situation, however, the relative error has little influence on the heat source estimate since the latter mainly depends on the temperature derivative.

\subsection{Checking the heat source computation}

The reliability of the heat source derivation must also be checked. To illustrate the capabilities of the image processing, a direct 1D (resp. 2D) thermal problem is first solved for a given 1D (resp. 2D) distribution of dissipative and thermoelastic sources. Naturally, initial and boundary conditions also have to be fixed. Because of small temperature variations, linear Fourier conditions were systematically chosen to model heat exchanges between the sample and the surrounding air and between the sample and the grips of the testing machine. The temperature field, as determined by the solution of the (direct) partial derivative problem, is finally used to test the image processing method (inverse problem), with the reconstructed distribution of heat sources being compared with the given initial one.

In Figs. 4 and 5, a 1D example was proposed. The evolution of the thermoprofile was derived from the following parameters: the heat sources were randomly distributed in space and constant in time. The thermoelastic source amplitude and that of the dissipation intensity varied within $(100 \pm 5){ }^{\circ} \mathrm{C} \mathrm{s}^{-1}$ and $(0.45 \pm 0.2){ }^{\circ} \mathrm{C} \mathrm{s}^{-1}$, respectively. This should reproduce the heterogeneous character of the fatigue process and its slow evolution during the test for fixed loading conditions. We superimposed Gaussian white noise in accordance with the analysis of the thermosignal given by the camera (crest amplitude $0.05^{\circ} \mathrm{C}$ ).

Variations in the thermoprofile are plotted in Fig. 4. The loading frequency $f_{\mathrm{L}}$ and sampling frequency $f_{\mathrm{S}}$ were, respectively, $50 \mathrm{~Hz}$ and $19 \mathrm{~Hz}$. Both values correspond to standard loading and sampling conditions used during the camera shots.

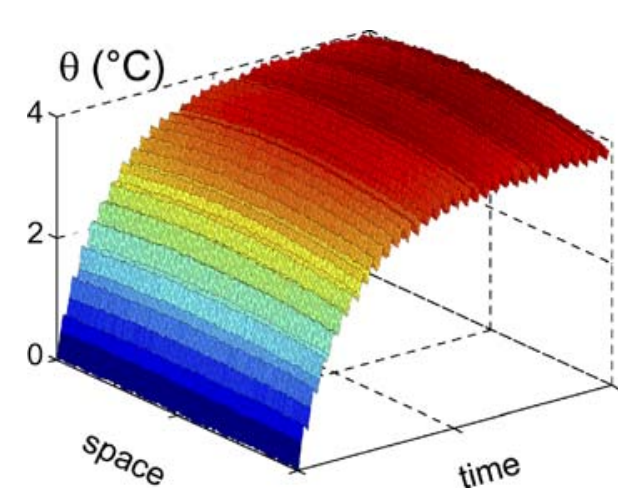

Fig. 4. Variations in the noisy longitudinal thermoprofile $\left(f_{\mathrm{S}}=19 \mathrm{~Hz}\right)$. 

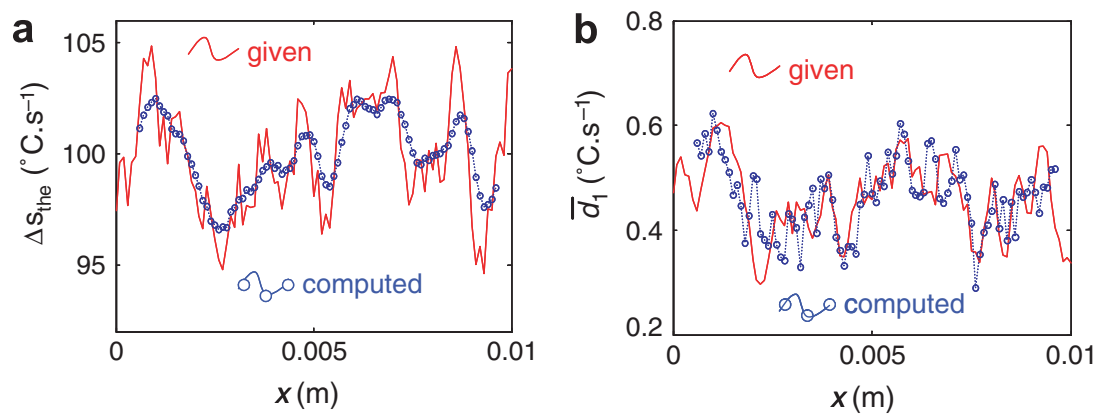

Fig. 5. Comparison between given and computed values: (a) thermoelastic source range; (b) dissipative source.
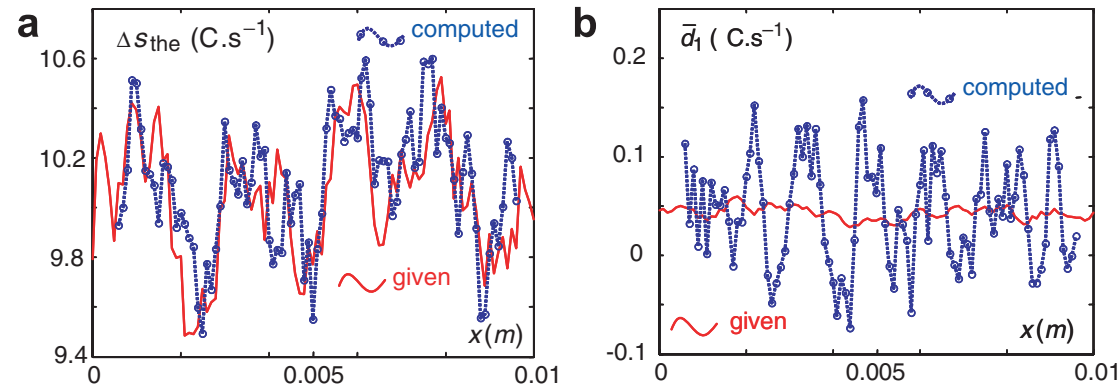

Fig. 6. Comparison between given and computed values: (a) thermoelastic source range; (b) dissipative source.

Fig. 4 shows small truncated (under-sampled) oscillations due to the thermoelasticity superimposed on a thermal drift due to dissipation. Progressive saturation of the drift is induced by the heat losses.

The reliability of the data processing was checked by comparing the random heat source distribution with that resulting from the data processing in the case of a noisy, under-sampled thermal signal.

A comparison between the given and computed heat source values is given in Fig. 5(a). We plotted the mean profiles of $\Delta s_{\text {the }}$ and $\bar{d}_{1}$ over the time period chosen for this numerical test (around $50 \mathrm{~s}$ ). Besides, the sources were divided by $\rho C$ and then expressed in ${ }^{\circ} \mathrm{C} \mathrm{s}^{-1}$. This operation makes it possible to define, for each type of source, an equivalent heating speed associated with a monotonous thermal process in adiabatic conditions and facilitates comparison between the coupling and dissipative sources.

Despite the different orders of magnitude of both source types, the data processing was able to correctly extract the weak dissipation intensity (around $0.5^{\circ} \mathrm{C} \mathrm{s}^{-1}$ ). The method may naturally become unstable and fail when there is lower dissipation intensity or thermal noise of greater crest amplitude. To illustrate these limits, we tested the data processing with the same previous conditions, but the source intensities were divided by 10. Fig. 6(a) shows that the data processing still computes correct thermoelastic source amplitudes. Nevertheless, the method gives corrupted dissipation amounts, as indicated in Fig. 6(b), and some values are even negative, which is physically unacceptable.
Note, however, that if we compute the average values of the given and computed dissipation profiles, we find $\bar{d}_{1}^{\mathrm{g}}=0.0428{ }^{\circ} \mathrm{Cs}^{-1}$ and $\bar{d}_{1}^{\mathrm{c}}=0.0431^{\circ} \mathrm{Cs}^{-1}$, respectively. This result shows that, for very low dissipation levels, the current method can be used to compute at best the mean dissipation over the sample gauge part.

\section{Experimental results}

\subsection{Thermoelasticity: profile analysis}

The 1D data processing enabled us to evaluate the time course of the longitudinal profile of $\Delta s_{\text {the }}$ related to the fatigue of the 2024 T3 aluminium alloy. In Fig. 7, the thermoelastic source range (expressed in ${ }^{\circ} \mathrm{C} \mathrm{s}^{-1}$ ) was plotted using a space-time representation (1 pixel approximately equal to $0.2 \mathrm{~mm}$ ). The corresponding $\sigma_{\mathrm{a}}$ and $R_{\sigma}$ were, respectively, $27 \mathrm{MPa}$ and 0.1 .

The coupling thermoelastic source distribution is here associated with one of the first steps of the fatigue test under axial loading. At this level, the number of cycles at low stress amplitude endured by the specimen is still low (around 50,000 cycles). Nevertheless, a slight heterogeneity in the thermoelastic source distribution can already be detected whereas a uniform distribution should be observed for a homogeneous uniaxial pulsating test. Moreover, the places where the ranges of the thermoelastic sources were the most important remained fixed from one block to another. By using 2D image processing, we stress 


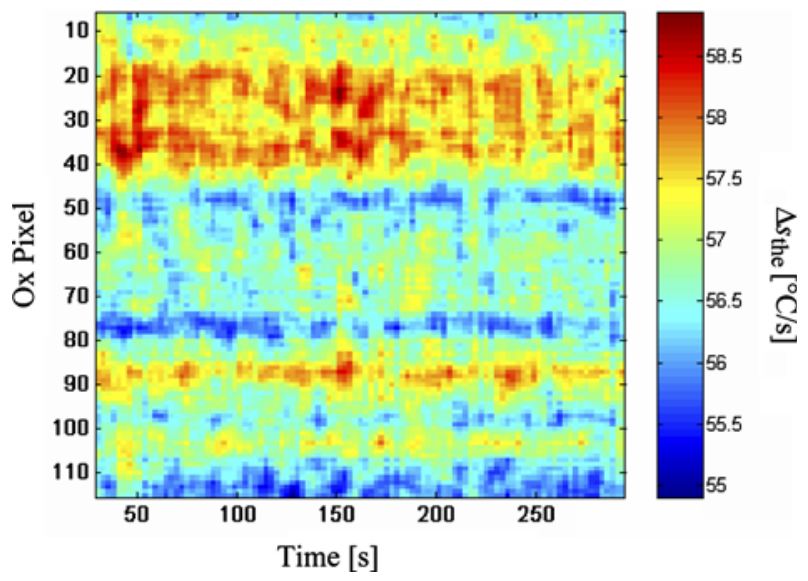

Fig. 7. Time course of the profile of the thermoelastic source range $\Delta s_{\text {the }}$ during the third loading block.

hereafter that these candidate zones may represent potential crack onset loci.

\subsection{Thermoelasticity: image analysis}

The application of 2D image processing confirms the presence of zones where the coupling source is greater since the first step of the test. The space distributions of $\Delta s_{\text {the }}$ relative to loading block \#1 and \#15 are plotted in Fig. 8(a) and (b).

Use of the 2D approach naturally gives more precise spatial detection of critical zones. In Fig. 8(a) and (b), we can observe in particular that in the region centered on $[0,5] \times[95,105]$ the amplitude of $\Delta s_{\text {the }}$ is highest from block $\# 1$ to the beginning of block \#15.

This crack occurred at the middle of block \#15 (left side, pixel 103) and propagated widthwise. We observed a sharp localization of thermoelastic sources in accordance with the stress concentration at the crack tip.

Fig. 9(a) shows the effects of the crack on the thermoelastic source distribution. In Fig. 9(b) the trend of thermoelastic sources at failure section is pointed out during a series of 300 cycles. The displacement of the maximum distribution profile corresponded to the crack propagation.

\subsection{Dissipation: first global assessments}

No reliable results have been obtained so far concerning 2D and even 1D distributions of dissipation because of the bad signal-to-noise ratio. The thermal noise is too high to correctly extract the dissipation from the thermosignal. This current limit of the calorimetric approach is directly

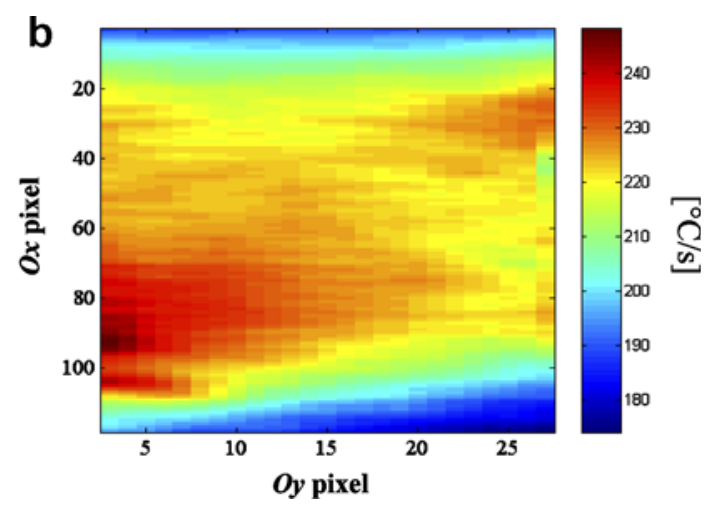

Fig. 8. Two-dimensional distribution of $\Delta s_{\text {the }}\left(f_{\mathrm{L}}=50 \mathrm{~Hz}, f_{\mathrm{S}}=250 \mathrm{~Hz}\right)$ : (a) loading block \#1; (b) loading block \#15.

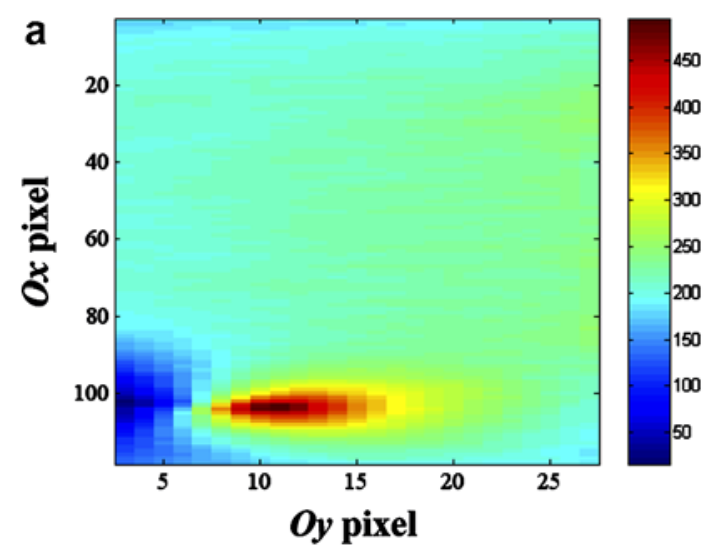

Fig. 9. (a) Two-dimensional distributions of $\Delta s_{\text {the }}\left(f_{\mathrm{L}}=50 \mathrm{~Hz}, f_{\mathrm{S}}=250 \mathrm{~Hz}\right)$ : crack propagation. (b) Variations in the $\Delta s_{\text {the }}$ profile at the failure section during 300 cycles. 
due to the tested aluminium alloy (low dissipation level, high thermal diffusivity) [11]. Note that for other alloys (like steels), the method has already given satisfactory results, even for the 2D approach $[12,13]$.

As with the second numerical example, we computed the dissipation corresponding to the whole specimen gauge part for the different stress ranges of the 15 blocks. We compared these mean dissipations with those associated with the energy of the hysteresis area during the cyclic stress-strain response. The hysteresis areas were estimated using the extensometer and load cell signals at low loading frequency.

In Fig. 10, the mechanical estimate of the mean dissipation per cycle was plotted during a fatigue test performed at $f_{\mathrm{L}}=1 \mathrm{~Hz}$ for a sampling frequency of $f_{\mathrm{S}}=50 \mathrm{~Hz}$.

Comparing mechanical and thermal estimates of dissipation is quite problematic for aluminium because the clamping system of the extensometer becomes unstable at high $f_{\mathrm{L}}$, while at low $f_{\mathrm{L}}$ the thermal drift is essentially due to warming of the testing machine. To offset this problem, the dissipation property has been studied by testing several small loading frequencies.

In Fig. 11, the mean dissipation per cycle divided by the loading frequency were reported for two fatigue tests performed at $f_{\mathrm{L}}=1 \mathrm{~Hz}$ and $f_{\mathrm{L}}=0.5 \mathrm{~Hz}$ (with $f_{\mathrm{S}}=25 \mathrm{~Hz}$ ), respectively.

This figure clearly shows that dissipation is proportional to $f_{\mathrm{L}}$. From a thermodynamic viewpoint, this result is interesting to qualify the irreversibility accompanying the fatigue test. The mechanical results obtained at low frequencies are in agreement with the dissipation model introduced in plasticity theory even though the stress level is obviously under the macroscopic yield stress.

If this dissipation property is now assumed for higher loading frequencies, it is possible to compare the mechanically predicted values of dissipation at $f_{\mathrm{L}}=50 \mathrm{~Hz}$ with those obtained thermally.

Fig. 12 shows this comparison. The data are scattered at low stress ranges. Conversely, the comparison is quite good for stress ranges greater than $60 \mathrm{MPa}$. The divergence at

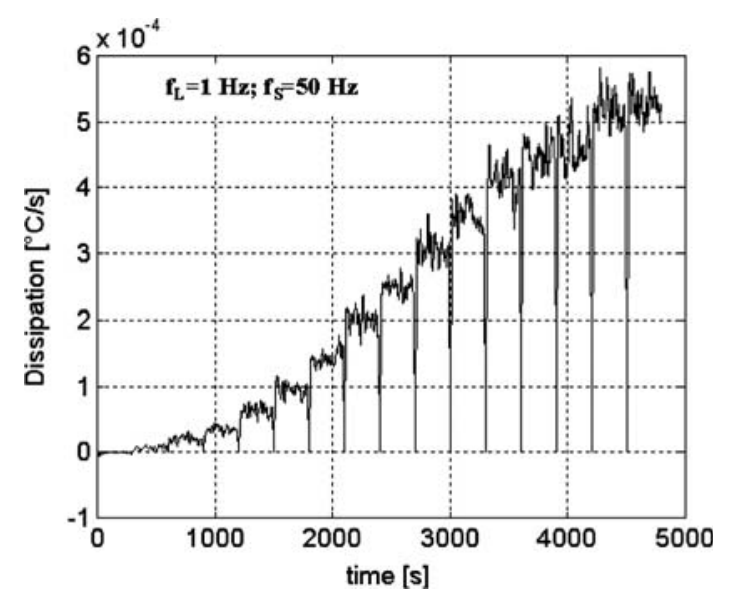

Fig. 10. Estimates of $d_{1} / \rho C$ via hysteresis area measurements.

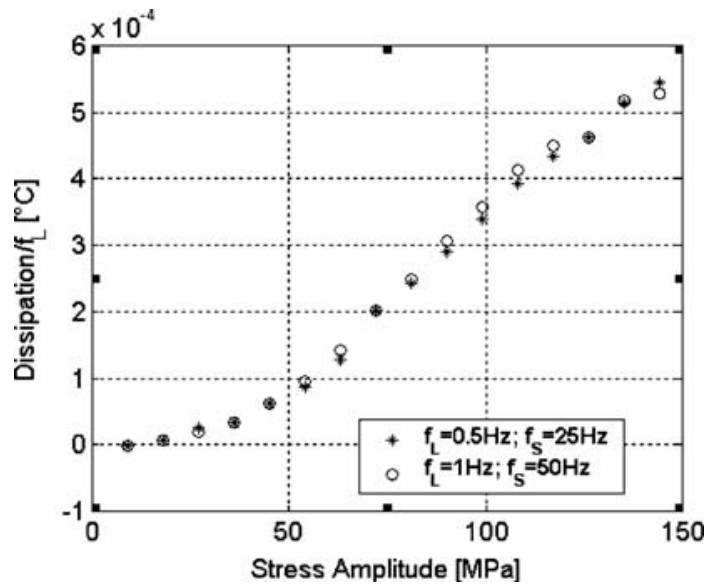

Fig. 11. Estimates of $d_{1} / \rho C / f_{\mathrm{L}}$ for two loading frequencies.

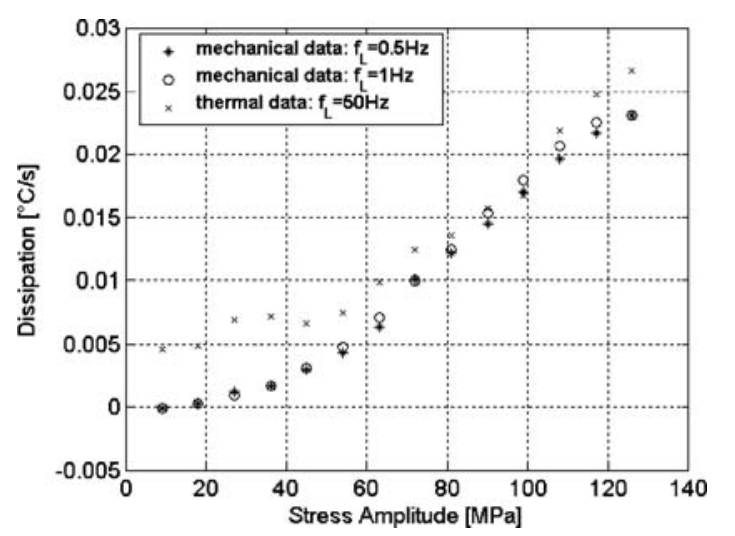

Fig. 12. Comparison of thermal and mechanical estimates of $d_{1} / \rho C$.

low stress levels is not surprising considering that the thermal drift of the testing machine induces a bad signal-tonoise ratio.

\section{Concluding comments}

In this paper, we presented an infrared image processing method that enabled us to estimate the different coupling and dissipative sources accompanying fatigue of the 2024 T3 aluminium alloy. Therefore a local expression of the heat equation was used. The frequency spectra of the thermoelastic amplitude and of the mean dissipation per cycle enabled us to compute both sources with the same thermosignal. As expected, the sensitivity of the method strongly depended on the intensity and distribution of the investigated heat sources and then on the signal-to-noise ratio of the temperature measurements. Several sub-cases were thus successively explored: the 2D method directly uses the thermal image supplied by an IR camera and computes the surface distribution of depthwise averaged sources; the 
1D method uses widthwise averaged thermal images and then derives (longitudinal) heat source profiles. Numerical tests were shown to illustrate the capabilities and limits of the calorimetric approach. In particular for low dissipation levels $\left(<0.05^{\circ} \mathrm{C} \mathrm{s}^{-1}\right)$ and for standard thermal noise crest amplitudes $\left(0.05^{\circ} \mathrm{C}\right)$, global amounts of dissipation were estimated at best.

Applications to the $2024 \mathrm{~T} 3$ aluminium alloy gave 1D and 2D heterogeneous thermoelastic source amplitude distributions. These heterogeneities may be attributed to the loading conditions or to the heterogeneity of the specimen. Whatever the origin, the localized zones, where the amplitude was more important, remained the same from one block to another. Moreover, we observed that these zones were potential loci of fatigue crack inception. The thermoelastic source profile associated with the cross-section where the crack occurs may be used to track crack propagation.

The low dissipation levels of the $2024 \mathrm{~T} 3$ aluminium and its high thermal diffusivity did not allow us to show reliable $2 \mathrm{D}$ or $1 \mathrm{D}$ dissipation distributions. We were then led to compute the average dissipation for the entire specimen. For the 15 blocks of the fatigue test, we also derived the mean dissipation from the computation of the mechanical hysteresis area. These comparisons were possible by extending, to high frequencies, the linear dependence of the dissipation with respect to $f_{\mathrm{L}}$. For stress amplitudes greater than $60 \mathrm{MPa}$ at $50 \mathrm{~Hz}$, we noted close agreement between the mechanical and thermal dissipation estimates. Prompted by these first encouraging results, our next objective is to improve the signal-to-noise ratio of the thermal images in order to be able to build 1D or even 2D dissipation distributions.

\section{References}

[1] Luong MP. Fatigue limit evaluation of metals using an infrared thermographic technique. Mech Mater 1998;28:155-63.

[2] La Rosa G, Risitano A. Thermographic methodology for rapid determination of the fatigue limit of materials and mechanical components. Int J Fatigue 2000;22(1):65-73.

[3] Liaw YB, Wang PK, Jiang L, Huang JY, Kuo RC, Huang JC. Thermographic investigation of the fatigue behaviour of reactor pressure vessel steels. Mater Sci Eng A 2001;314:131-9.

[4] Krapez JC, Pacou D, Gardette G. Lock-in thermography and fatigue limit of metals. Quantitative infrared thermography 5. In: Balageas D, Beaudoin JL, Busse G, Carlomagno GM, editors. Proceedings of QIRT'2000, 2000. p. 277-82.

[5] Cugy P, Galtier A. Microplasticity and temperature increase in low carbon steels. In: Proceedings of the 8 th international fatigue conference, 2002. p. 549-56.

[6] Mabru C, Chrysochoos A. Dissipation et couplages accompagnant la fatigue des matériaux, métalliques. In: Berthaud et al., editors. Proceedings of photomécanique'01, 2001. p. 375-82.

[7] Germain P, Nguyen QS, Suquet P. Continuum thermodynamics. J Appl Mech 1983;50:1010-20.

[8] Capatina A, Stavre R. Algorithms and convergence results for an inverse problem in heat propagation. Int J Eng Sci 2000;38:575-87.

[9] Chrysochoos A. Analyse du comportement des matériaux par thermographie infrarouge. In: Berthaud et al., editors. Proceedings of photomécanique'95, 1995. p. 203-11.

[10] Chrysochoos A, Louche H. An infrared image processing to analyse the calorific effects accompanying strain localisation. Int J Eng Sci 2000;38:1759-88.

[11] Morabito AE. Analisi termomeccanica degli effeti termoelastici e dissipativi associati al comportamento a fatica della lega di alluminio 2024 T3. PhD thesis, Universita'degli Studi di Lecce, 2003.

[12] Chrysochoos A, Boulanger T, Wattrisse B, Morabito AE. Analyse calorimétrique de la fatigue des métaux. In: Proceedings of photomécanique'04, 2004. p. 401-08.

[13] Boulanger T, Chrysochoos A, Mabru C, Galtier A. Calorimetric and thermoelastic effects associated with the fatigue behavior of steels. Int J Fatigue 2004;26:221-9. 